BMJ Open Sport \& Exercise Medicine

\title{
Novel insights of overtraining syndrome discovered from the EROS study
}

\author{
Flavio A Cadegiani, ${ }^{\circledR}$ Claudio Elias Kater
}

To cite: Cadegiani FA, Kater CE. Novel insights of overtraining syndrome discovered from the EROS study. BMJ Open Sport \& Exercise Medicine 2019;5:e000542. doi:10.1136/ bmjsem-2019-000542

The results of the study are presented clearly, honestly and without fabrication, falsification or inappropriate data manipulation. Raw data can be obtained from the repository mentioned in the study.

Accepted 20 May 2019
Check for updates

(C) Author(s) (or their employer(s)) 2019. Re-use permitted under CC BY-NC. No commercial re-use. See rights and permissions. Published by BMJ

Endocrinology, Universidade Federal de Sao Paulo, Sao Paulo, Brazil

Correspondence to Dr Flavio A Cadegiani; superendocrinology@gmail.com

\section{ABSTRACT}

Background Excessive training and inadequate recovery could cause 'overtraining syndrome' (OTS), which is characterised by underperformance and fatigue. The pathophysiology of OTS is unclear. We aimed to describe novel mechanisms and risk factors associated with OTS, and thereby facilitate its early identification and prevention, from a comprehensive joint qualitative analysis of the findings from all the four arms of the Endocrine and Metabolic Responses on Overtraining Syndrome (EROS) study.

Methods We compared the types and proportions of behavioural patterns of 67 evaluated parameters of OTS from 51 participants-athletes with OTS (OTS, $n=14$ ), healthy athletes $(n=25)$ and healthy non-physically active controls $(n=12)$. We performed overall and pairwise comparisons for statistically significant differences between the three groups $(p<0.05)$.

Results A total of 44 (65.7\%) markers exhibited significant differences between the three groups: 32 $(72.7 \%)$ showed a loss of the conditioning effect of exercise ('deconditioning'), 7 (15.9\%) showed changes exclusive to 0TS, $3(6.8 \%)$ maintained the exerciseinduced conditioning effects and $2(4.5 \%)$ revealed an exacerbation of the adaptive changes to exercises. Conclusion Our findings suggest that OTS is likely triggered by multiple factors, not restricted to excessive training, resulted from a chronic energy deprivation, leading to multiple losses in the conditioning processes typically observed in healthy athletes, as a combination of 'paradoxical deconditioning' processes, which explains the gradual and marked loss of physical conditioning found in OTS. We, therefore, suggest that the term 'paradoxical deconditioning syndrome' better represents the features of this syndrome.

\section{INTRODUCTION}

Complex diagnostic criteria, triggers and pathophysiology make the diagnosis of OTS challenging. ${ }^{12345}$ Its estimated prevalence varies widely $(15 \%-60 \%)$, and depends on the frequency, intensity of training and type of sport. ${ }^{167}$ Potential biomarkers of OTS include higher creatine kinase (CK), lower postexercise lactate, and blunted exercise-stimulated prolactin, growth hormone $(\mathrm{GH})$, cortisol and adrenocorticotropic hormone (ACTH)

\section{What are the new findings?}

Among 67 metabolic, hormonal, biochemical, and clinical parameters compared between sex, age, and BMI-matched groups of athletes affected by actual overtraining syndrome (OTS), healthy athletes (ATL), and non-physically active subjects (NPAC), 44 $(65.7 \%)$ had at least one sort of change in ATL, OTS or both, of which $26(59.1 \%)$ were found to be different in ATL, while similar between OTS and NPAC.

- Dysfunctions in OTS were neither overt nor absent, but 'relatively altered', as the majority of the parameters were altered when compared to healthy athletes, but normal when compared to the reference ranges for general population.

- OTS results from a mix of losses of the multiple biochemical and hormonal conditioning processes found to occur in athletes, which we termed as 'mix of deconditioning process'.

- OTS occurred independently from excessive training, since insufficient calorie, protein, or carbohydrate intake, poor sleep quality, or concurrent excessive cognitive effort were the found to be prevailing predictors of OTS.

- The understanding of OTS as a combination of 'deconditioning processes' helps to elucidate the underlying reason of the unexplained decrease in performance, the hallmark of OTS.

- We found sufficient data to suggest "Paradoxical Deconditioning Syndrome" as a more appropriate and descriptive name for OTS than the previous misnomer ('overtraining'). Further studies are needed to confirm the novel mechanisms of OTS revealed by the EROS study.

- The EROS study also unveiled the existence of multiple independent metabolic and hormonal adaptations to exercise, in addition to those in cardiovascular, autonomic, and neuromuscular systems. This serendipitous finding may explain some of the health benefits and progressive improvement observed in athletes.

responses. $^{128-18}$ Basal hormones and other biochemical parameters are normal in OTS. ${ }^{16-18}$ There is currently no conclusive data on the causes, pathophysiology, early identification and prevention of OTS, ${ }^{1} 21019$ ${ }^{20}$ as reinforced by a systematic review. ${ }^{10}$ 
We designed the Endocrine and Metabolic Responses on Overtraining Syndrome (EROS) study ${ }^{21-24}$ to try to elucidate the pathophysiology of OTS, identify new biomarkers and risk factors, and propose new tools for prevention and early diagnosis of OTS. We evaluated and compared clinical, biochemical and metabolic changes in athletes with OTS (OTS group), age-matched, body mass index (BMI)-matched and sex-matched healthy athletes (ATL group) and non-physically active control subjects (NPAC group). The inclusion of a second control group of non-athletes provided a context in which to interpret differences between OTS-affected athletes and healthy athletes. The simultaneous comparison of healthy athletes and non-athletes enhanced our understanding of the differences between the OTS-affected and healthy athletes, that is, differences that were also present when OTS-affected athletes were compared with non-athletes as a reference group, and differences between the OTS-affected and healthy athletes that were not present when the OTS-affected athletes were compared with non-athletes. Relative differences refer to the analysed parameters that were different from those typical of athletes, but not different from the general population.

The parameters evaluated in the EROS study revealed approximately 45 new markers in both the healthy and OTS-affected athletes. ${ }^{21-24}$ We hypothesised that levels of dysfunction in OTS vary widely, depending on the parameters evaluated, as we found different types of behaviours in OTS-affected athletes compared with healthy athletes and non-athletes, which could help to predict those at high risk for OTS.

First, the behaviours referred to as unaltered markers were similar for both the healthy athletes and non-athletes. Second, the markers were similar to those of healthy athletes, and different from those of non-athletes indicating the adaptation of these parameters to physical activity, which was maintained in OTS. Markers that disclosed these two types of behaviours were not helpful to detect imminent OTS.

Third, the markers were different from those of healthy athletes, but were similar to those of healthy non-athletes, while the markers of healthy athletes were different from those of non-athletes, indicating these parameters underwent adaptive changes to exercise, which seemed to be absent in the presence of OTS, likely as a loss of these adaptive processes. Fourth, the markers were different from those of healthy athletes, which were also different from those of non-athletes. This finding suggests the physiological adaptation to exercise was exacerbated in OTS with higher marker levels in healthy athletes than non-athletes, and even higher marker levels in OTS-affected athletes. It also may indicate a loss of adaptation to exercise, leading to overt dysfunction (ie, higher marker levels were found in healthy athletes than non-athletes, which were higher than those of OTS-affected athletes were). Parameters from the third or fourth types of behaviours were those that were potentially valuable for the detection of OTS.
The objective of this study was to identify new insights for OTS, by conducting a comprehensive joint analysis of data from the four arms of the EROS study (EROS-HPA axis, EROS-STRESS, EROS-PROFILE and EROSBASAL). ${ }^{21-24}$

We set out to clarify the behaviours of each parameter, the predominant types of behaviours in OTS-affected athletes, the metabolic and biochemical changes caused by athletic training and their disruptions in OTS, and the metabolic and hormonal changes caused by OTS through post hoc analyses.

On the basis of these findings, we aimed to hypothesise novel risk factors for OTS, to elaborate predictive models to detect athletes at high risk or imminent OTS for an effective preventive approach and early identification of OTS, and to hypothesise novel mechanisms that underlie the pathophysiology of OTS, to be further elucidated.

\section{METHODS}

To avoid repetition of previously reported material and methods, ${ }^{21-24}$ we have summarised the recruitment, selection and procedures performed. The detailed design, methods, participant selection criteria and baseline characteristics of the EROS study are available in the previously published papers and at a repository (https:/ osf.io/bhpq9). The complete raw data, including individual results, are located in the same repository address.

\section{Participant's selection}

We recruited participants through sports coaches, social media (Facebook and Instagram), group messages (WhatsApp and Telegram) and organic spread. Each candidate provided information regarding age, sex, estimated weight and height, and the group they intended to participate in. To prevent bias, specific criteria for OTS, ATL and NPAC groups were required for all subjects, as presented in table 1. We avoided extremes of age, false athletes, false sedentary, misdiagnosis of OTS, use of confounding drugs or hormones and presence of confounding diseases by using a two-step process; the first step excluded participants based on medical history and physical examination, and the second step was a basic biochemical evaluation for confounding alterations.

Of the 117 markers evaluated in the EROS study, we excluded those that by themselves did not determine diagnoses or status (16 markers), qualitative indices (9 markers), markers that were intrinsically linked to other parameters (7 markers), markers that did not provide additional independent data (2 markers), markers that were possible triggers of OTS and not the behavioural consequences of exercise (4 markers), invalid and/or unsubstantiated data (9 markers) and markers that were missing in more than $5 \%$ of the participants (3 markers), for a total of 50 excluded markers (table 2).

We eventually evaluated 67 parameters, including hormonal responses to stimulation tests, basal and accumulated hormonal levels, social and psychological 
Table 1 Clinical inclusion criteria for the EROS study

\begin{tabular}{lll}
\hline All participants & All athletes & OTS-affected athletes \\
\hline $\begin{array}{l}\text { 1. Male sex, 18-50 y/o, 20-32.9 } \\
\mathrm{kg} / \mathrm{m}^{2} \text { (athletes) and 20-29.9 } \\
\mathrm{kg} / \mathrm{m}^{2} \text { (sedentary) }\end{array}$ & $\begin{array}{l}\text { 1. Weekly exercise at least four } \\
\text { times and }>300 \text { min of moderate-to- } \\
\text { vigorous training intensity }\end{array}$ & $\begin{array}{l}\text { 1. Underperformance of } \geq 10 \% \text { of previous } \\
\text { performance as verified by certified sports coach, or } \\
\text { loss of } \geq 20 \% \text { in time-to-fatigue, with self-reported } \\
\text { increase in sense of effort in training relative to before } \\
\text { OTS }\end{array}$ \\
$\begin{array}{lll}\text { 2. No previous psychiatric } \\
\text { disorders or use of centrally } \\
\text { acting drugs }\end{array}$ & $\begin{array}{l}\text { 2. Continuous training for at least } \\
\text { 6 months with no interruption of } \\
\text { training for }>30 \text { days }\end{array}$ & $\begin{array}{l}\text { 2. Prolonged underperformance not explained by } \\
\text { conditions that could lead to decrease in performance, } \\
\text { such as infections, inflammation, actual primary } \\
\text { hormonal dysfunctions, psychosocial or psychiatric } \\
\text { conditions, or emotional and social problems }\end{array}$ \\
$\begin{array}{ll}\text { 3. No hormonal therapy in the } \\
\text { preceding 6 months }\end{array}$ & $\begin{array}{l}\text { 3. Persistent fatigue (>2 weeks), as a subjective feeling, } \\
\text { further confirmed by the Profile of Mood Scales, and } \\
\text { decreased sleep quality (self-reported, compared with } \\
\text { previous sleep quality) }\end{array}$ \\
\hline
\end{tabular}

EROS, Endocrine and Metabolic Responses on Overtraining Syndrome; y/o, years old.

aspects, specific eating patterns, and body metabolism and composition, as detailed in table 3 .

Biochemical data were determined using standardised commercially available assay kits $^{21-24}$ (https://osf.io/ bhpq9). The interassay and intra-assay coefficients of variability of the biochemical markers measured in all arms of the EROS study were below $3.5 \%$ and $3 \%$, respectively.

\section{Classification of the parameters}

The means of all 67 parameters were analysed using one-way analysis of variance (ANOVA) for normally distributed parameters, and medians were analysed using the Kruskal-Wallis test (a non-parametric type of ANOVA) for non-normally distributed parameters. When $\mathrm{p}<0.05$, pairwise comparisons were performed using Dunnett's T3 and Tukey's post hoc analysis, and Dunn's post hoc adjusted test, for normally and non-normally distributed parameters, respectively.

From the similarities between pairwise comparisons between athletes with OTS and healthy athletes, healthy athletes and non-athletes, and athletes with OTS and non-athletes, we inferred possible scenarios for the behaviour of each parameter in healthy athletes relative to sedentary individuals, and in athletes with OTS, in comparison with healthy athletes and non-athletes. The possible scenarios or categories for each parameter enabled us to understand the patterns of each parameter in response to athletic training and in OTS. The possible scenarios and the specific criteria (figure 1) that we proposed were, defined as follows:

1. 'Unchanged marker': levels were similar in all groups, indicating that the parameter does not show any adaptive change to athletic training, or alterations in OTS.

2. 'Exclusive marker of OTS': levels were different in OTS, compared with ATL and NPAC, but similar between ATL and NPAC, indicating that the marker does not undergo any adaptive change in response to physical activity, but may participate in or result from the pathophysiology of OTS.
3. 'Maintained conditioning effect': ATL showed adaptive changes to exercise, compared with NPAC, and the adaptive changes were maintained in OTS (levels were similar between OTS and ATL). This showed that adaptive or conditioning changes in response to exercise were not lost during OTS.

4. 'Deconditioning': markers showed differences between ATL and NPAC, indicating an adaptive or conditioning effect to physical activity that was lost in OTS. In this scenario, the similarity between OTS and NPAC possibly indicates loss of adaptive changes to exercise, which we termed 'deconditioning'. We proposed additional subcategories (figure 2) for the deconditioning process, according to the level of loss of adaptive conditioning: (i) partial loss of adaptive change, or 'partial deconditioning', wherein levels in OTS were between those in ATL and NPAC and (ii) excessive loss, or 'exacerbated deconditioning', wherein differences between levels in OTS and ATL were greater than those in ATL and NPAC.

5. 'Exacerbated conditioning' or 'compensatory overconditioning': marker levels were higher in ATL than in NPAC, because of an adaptive or conditioning effect of regular training, and were even higher in OTS. This indicates that the conditioning effect to athletic training undergoes a compensatory exacerbation in OTS, possibly as a mechanism to maintain function despite energy deprivation.

To avoid bias in the classification into different scenarios, we pre-established cut-off criteria for the categories, as well as for exceptions. We examined statistical trends and identified exceptions that did not fit into the aforementioned categories; these were analysed individually. To discover possibly hidden markers for OTS, we considered markers non-similar whenever overall $p$ values were $>0.05$ but $<0.10$. We assumed that $\mathrm{p}<0.05$ was not always achieved owing to the relatively small number of subjects, and adherence to the strict 
Table 2 Markers evaluated by the EROS study that were not included in the present analysis

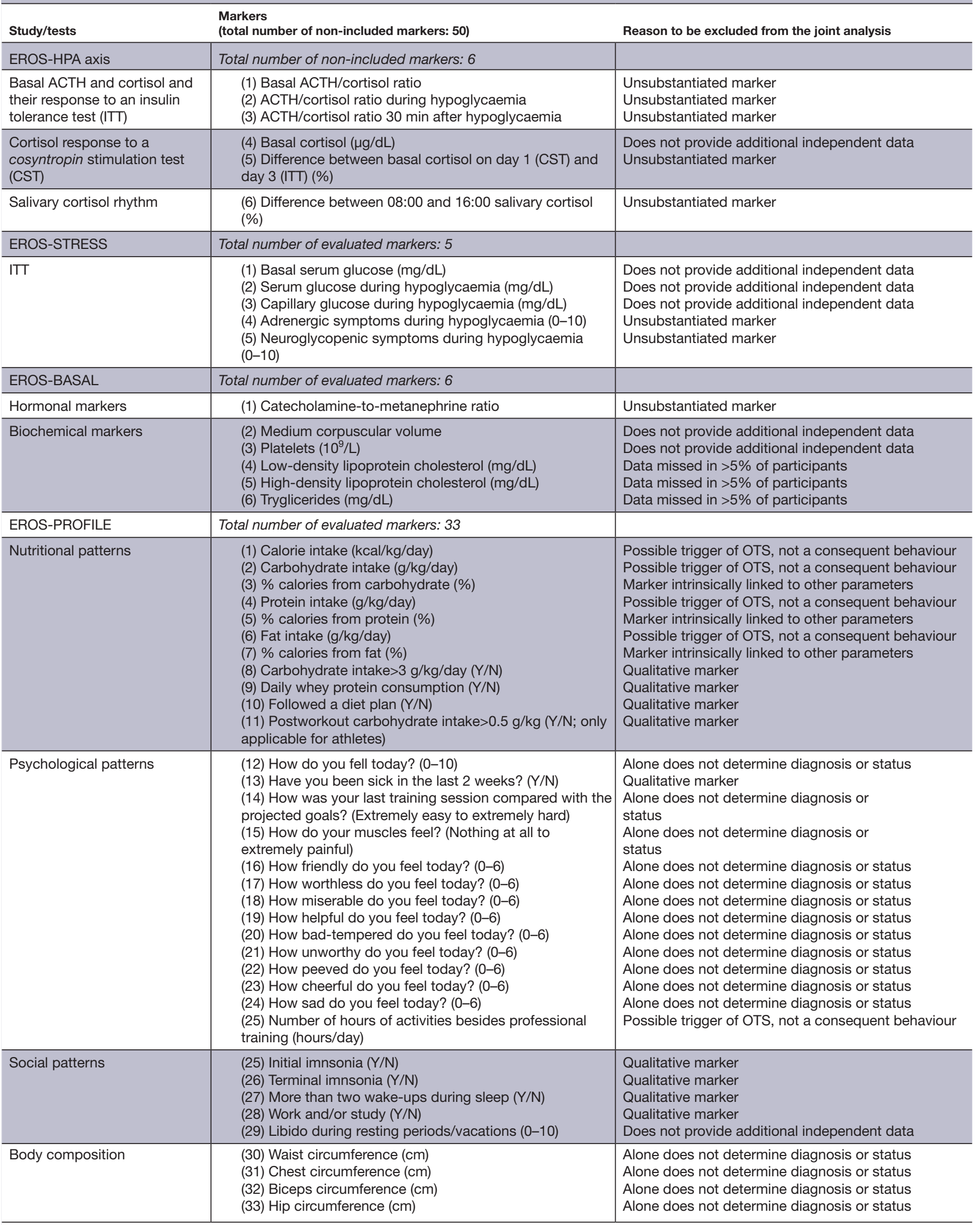

ACTH, adrenocorticotropic hormone; EROS, Endocrine and Metabolic Responses on Overtraining Syndrome; N, No; OTS, overtraining syndrome; Y, yes. 
Table 3 Markers evaluated by the EROS study and included in the present analysis (aside from inclusion criteria for all groups: body mass index, age and sex)

\begin{tabular}{ll}
\hline Study/tests & $\begin{array}{l}\text { Markers } \\
\text { (total number of markers: 67) }\end{array}$ \\
\hline EROS-HPA axis & Total number of evaluated markers: 15 \\
Basal ACTH and cortisol and their response to an insulin tolerance & (1) Basal ACTH (pg/mL) and (2) cortisol ( $\mu \mathrm{g} / \mathrm{dL})$ \\
test (ITT) & (3) ACTH and (4) cortisol during hypoglycaemia \\
& (5) ACTH and (6) cortisol 30 min after hypoglycaemia \\
& (7) ACTH and (8) cortisol increase during ITT
\end{tabular}

Cortisol response to a cosyntropin stimulation test (CST)

Salivary cortisol rhythm

EROS-STRESS

$\mathrm{GH}$ and prolactin response to an ITT

EROS-BASAL

Hormonal markers

(9) Cortisol at $30 \mathrm{~min}$ and (10) at $60 \mathrm{~min}$ after injection

(11) Salivary cortisol (ng/dL) at awakening and (12) 30 min after

(13) at $16: 00$ and (14) at 23:00

(15) Cortisol awakening response

Total number of evaluated markers: 7

(1) Basal $(\mathrm{GH})(\mu \mathrm{g} / \mathrm{L})$ and $(2)$ prolactin $(\mathrm{ng} / \mathrm{mL})$

(3) $\mathrm{GH}$ and (4) prolactin during hypoglycaemia

(5) $\mathrm{GH}$ and (6) prolactin 30 min after hypoglycaemia

(7) Prolactin increase during ITT

Total number of evaluated markers: 26

(1) Total testosterone (ng/dL) and (2) oestradiol $(\mathrm{pg} / \mathrm{mL})$

(3) IGF-1 (pg/mL), (4) TSH $(\mu \mathrm{Ul} / \mathrm{mL})$ and (5) free T3 $(\mathrm{pg} / \mathrm{mL})$

(6) Total catecholamines and (7) metanephrines (both $\mu \mathrm{g} / 12$ hours)

(8) Noradrenaline, (9) epinephrine and (10) dopamine (all $\mu \mathrm{g} / 12$ hours)

(11) Metanephrines and (12) normetanephrines (both $\mu \mathrm{g} / 12$ hours)

\begin{tabular}{|c|c|}
\hline Biochemical markers & $\begin{array}{l}\text { (13) Erythrocyte sedimentation rate }(\mathrm{mm} / \text { hour) and }(14) \text { haematocrit }(\%) \\
\text { (15) C reactive protein }(\mathrm{mg} / \mathrm{dL}) \text { and }(16) \text { lactate }(\mathrm{nmol} / \mathrm{L}) \\
\text { (17) Vitamin } B 12(\mathrm{pg} / \mathrm{mL}) \text { and }(18) \text { ferritin }(\mathrm{ng} / \mathrm{mL}) \\
\left.\text { (19) Neutrophils, }(20) \text { lymphocyte and }(21) \text { eosinophils (all } / \mathrm{mm}^{3}\right) \\
\text { (22) Creatine kinase }(\mathrm{U} / \mathrm{L})\end{array}$ \\
\hline Ratios & $\begin{array}{l}\text { (23) Testosterone-to-oestradiol and (24) testosterone-to-cortisol ratios } \\
\text { (25) Neutrophil-to-lymphocyte and (26) platelet-to-lymphocyte ratios }\end{array}$ \\
\hline EROS-PROFILE & tal number of evaluated markers: 19 \\
\hline General patterns & $\begin{array}{l}\text { (1) Duration of night sleep (hour) and (2) self-reported sleep quality (0-10) } \\
\text { (3) Self-reported libido }(0-10)\end{array}$ \\
\hline Psychological patterns & $\begin{array}{l}\text { (4) Profile of Mood State questionnaire (total score: }-32 \text { to }+120) \\
\text { (5) Anger }(0-48) \text { and }(6) \text { confusion }(0-28) \text { subscales } \\
\text { (7) Depression }(0-60) \text { and }(8) \text { vigour }(0-32) \text { subscales } \\
\text { (9) Fatigue }(0-28) \text { and (10) tension }(0-36) \text { subscales }\end{array}$ \\
\hline Body metabolism analysis & $\begin{array}{l}\text { (11) Measured-to-predicted basal metabolic rate (BMR, \%) } \\
\text { (12) Percentage of fat burning compared with total BMR (\%) }\end{array}$ \\
\hline Body composition & $\begin{array}{l}\text { (13) Body fat percentage (\%) and (14) muscle mass weight }(\mathrm{kg}) \\
\text { (15) Body water percentage (BW, \%) and (16) extracellular water compared with } \\
\text { total BW (\%) } \\
\text { (17) Visceral fat }\left(\mathrm{cm}^{2}\right) \text { and (18) waist circumference (cm) } \\
\text { (19) Chest-to-waist circumference }\end{array}$ \\
\hline
\end{tabular}

ACTH, adrenocorticotropic hormone; EROS, Endocrine and Metabolic Responses on Overtraining Syndrome; GH, growth hormone; IGF-1, Insulin-like Growth Factor 1; TSH, Thyroid Stimulating Hormone.

and correct diagnosis of OTS, which excluded almost $90 \%$ of the athletes with suspected OTS.

\section{Analysis of the scenarios}

Following classification, we analysed the behaviours of the 67 analysed parameters and calculated the percentages of parameters classified in each of the five scenarios, to estimate the predominant behaviours observed in OTS. On the basis of the predominant behaviours, we performed a descriptive analysis of the findings to develop a broader and deeper understanding of the syndrome, reinforce previously proposed theories on OTS, propose new hypotheses regarding novel pathophysiological pathways, detect new risk factors for OTS and build predictive models for the prevention and early diagnosis of OTS.

\section{RESULTS}

\section{Participants}

Of the 87 OTS, 46 ATL and 13 NPAC subjects initially identified during the recruitment process, 14 were selected for the OTS group, 25 for the ATL group and 12 for the NPAC group. The baseline characteristics (age, body weight and BMI) of the three groups were statistically similar. Supplementary information regarding the selection process and the baseline characteristics have been previously published. ${ }^{21-24}$ 


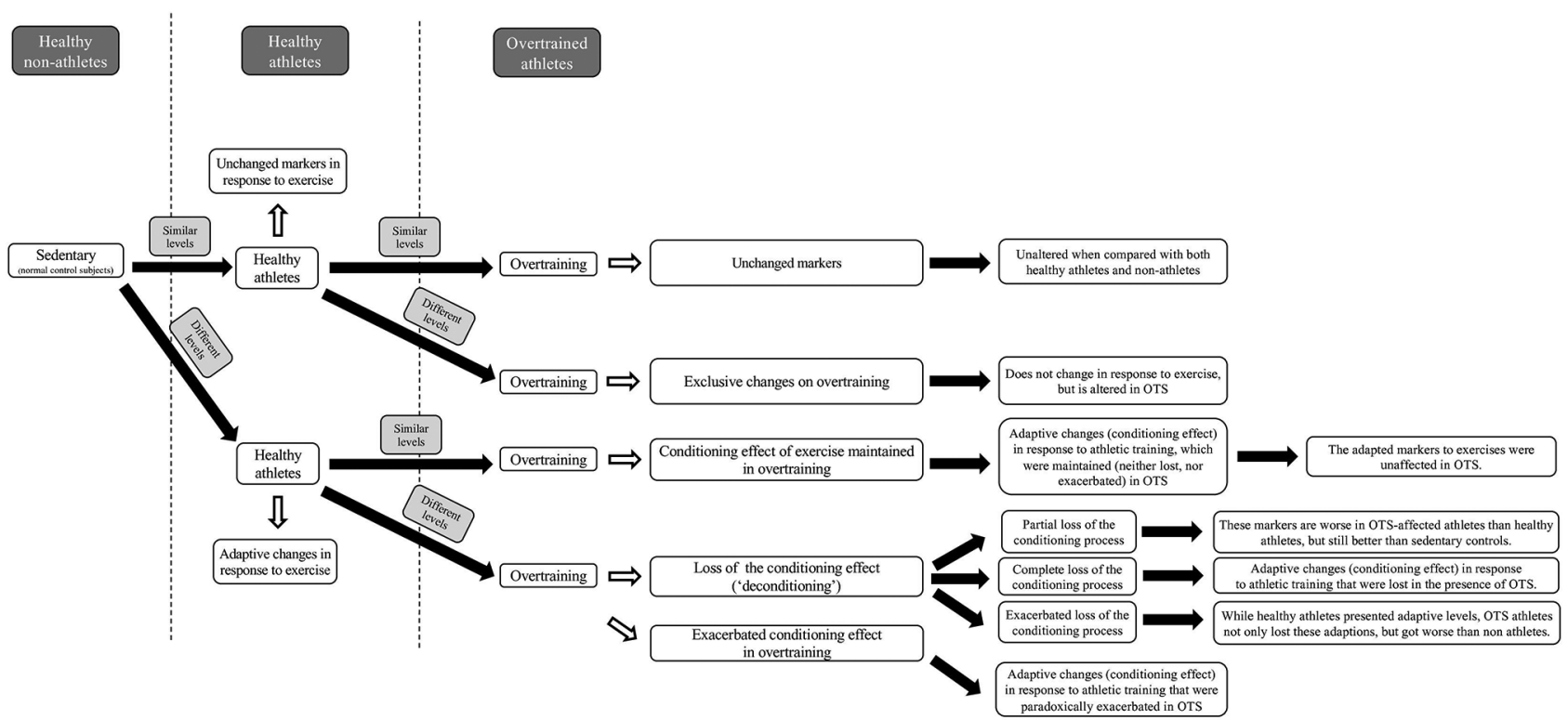

Figure 1 Behaviours of the evaluated parameters, according to the similarity between sedentary controls, healthy athletes and athletes affected by overtraining syndrome (OTS).

\section{Athletes with OTS}

The athletes with OTS were all diagnosed with true OTS, not FOR or NFOR, and were excluded for confounding diseases, as the average duration of fatigue was $44.3 \pm 23.0$ days, lasting for more than 2 weeks. None of these athletes had fully recovered by the time of the study, allowing the diagnosis of OTS and not over-reaching states, due to the prolonged decrease in performance. The sport modalities practiced by athletes with OTS were: high-intensity functional training (HIFT; $\mathrm{n}=11$;
78.6\%); cycling, long-distance running and weightlifting $(\mathrm{n}=1)$; middle-distance running and weightlifting $(\mathrm{n}=1)$; and swimming, cycling, weightlifting and karate $(n=1)$. The healthy athletes practiced: HIFT $(\mathrm{n}=24 ; 96 \%)$ and multiple sports $(n=1)$. All athletes from both the groups practised both the endurance and resistance exercises.

\section{Parameters classified into each scenario}

The classification of the 67 parameters into each hypothesised scenario, and the number of parameters in each
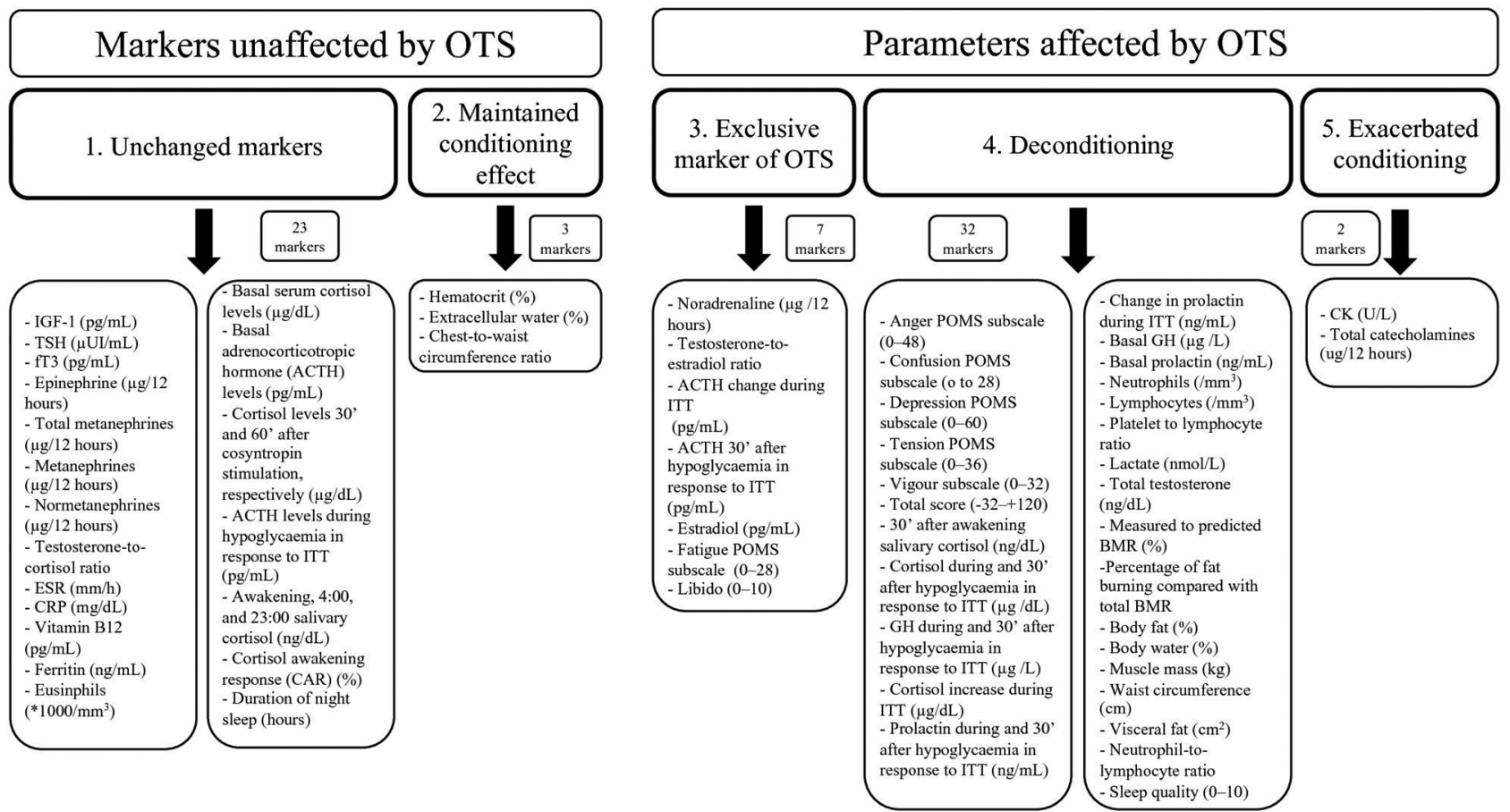

$\mathrm{BMR}=$ Basal metabolic rate $\mathrm{CK}=$ Creatine kinase $; \mathrm{CRP}=\mathrm{C}$ reactive protein $; \mathrm{ESR}=$ Erythrocyte sedimentation rate; ITT = Insulin tolerance test;; POMS = Profile of Mood States.

Figure 2 Markers according to each scenario. GH, growth hormone; fT3, free T3; OTS, overtraining syndrome. 


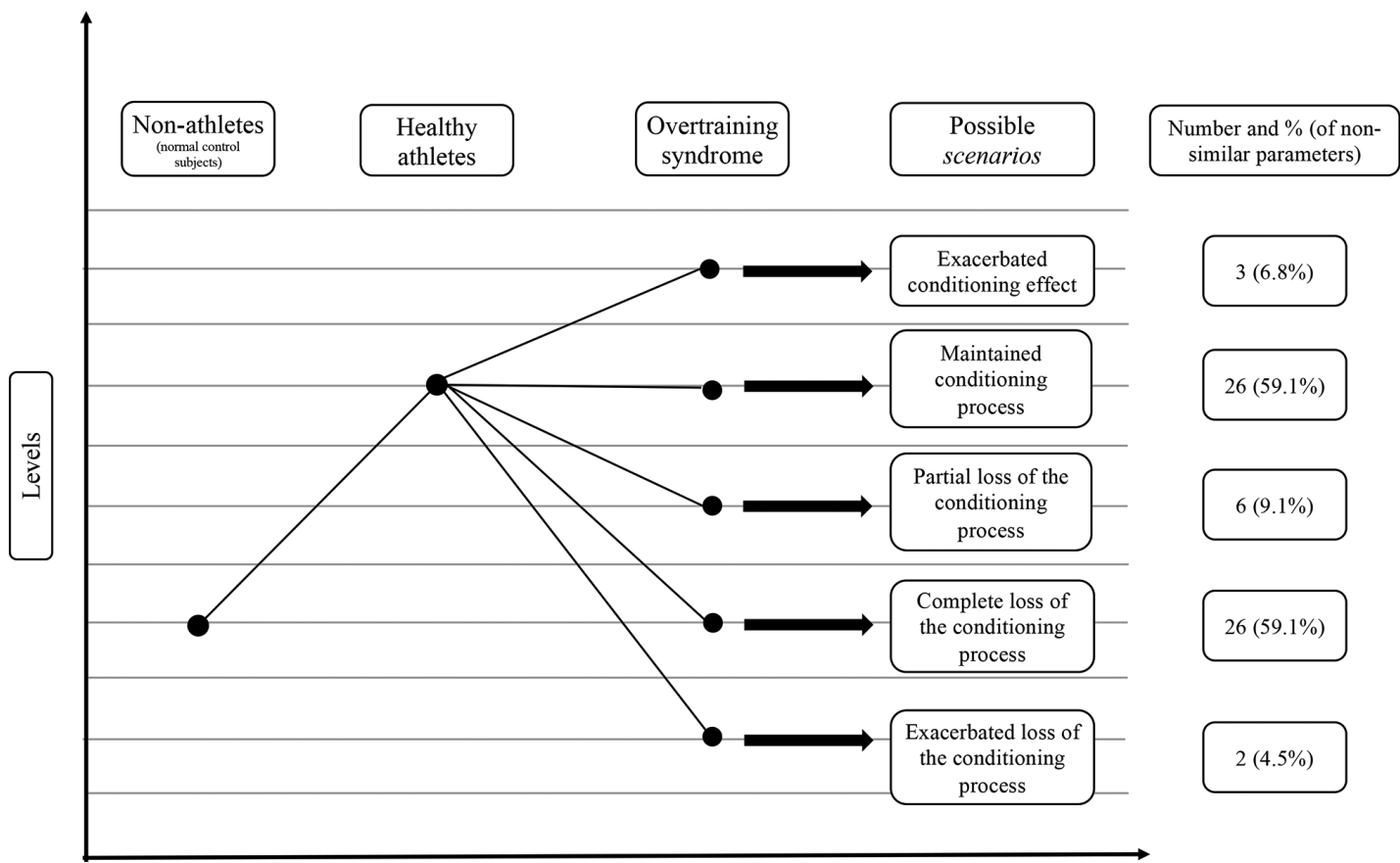

Figure 3 Behaviours of parameters in overtraining syndrome among those that demonstrated adaptive (conditioning) changes in response to athletic training.

scenario are detailed in figure 2. A total of 23 parameters $(34.3 \%)$ remained unchanged in the 3 groups (scenario \#1), while $44(65.7 \%)$ showed at least one significant difference between groups (scenarios \#2-\#5).

Of the total and non-similar markers, $4.5 \%$ and $6.8 \%$ (3 markers), respectively, showed sustained conditioning effects (scenario \#2); $10.4 \%$ and $15.9 \%$ (7 markers), respectively, showed exclusive OTS changes (scenario \#3); $47.8 \%$ and $72.7 \%$ (32 markers), respectively, exhibited loss of the conditioning processes observed in athletes, which we termed 'deconditioning processes' (scenario \#4); and 3.0\% and $4.5 \%$ (2 markers), respectively, exhibited exacerbation of adaptive changes (scenario \#5). Additionally, in the proposed subclassifications of the deconditioning processes (scenario \#4), according to the level of the loss of the adaptive conditioning process, $6.0 \%$ and $9.1 \%$ (4 markers) showed partial deconditioning (\#4a), 38.8\% and 59.1\% (26 markers) showed a complete loss of conditioning (\#4b), and $3.0 \%$ and $4.5 \%$ (2 markers) showed an exacerbation of the deconditioning process (\#4c). A summary of the non-similar markers is shown in figure 3 , while the biochemical markers found to be altered in OTS are described in table 4.

\section{DISCUSSION}

\section{Challenges in the assessment of OTS}

According to the latest guideline on OTS ${ }^{1}$ and a systematic review on the hormonal aspects of OTS, ${ }^{10}$ despite multiple studies and theories, the exact mechanisms underlying the complex pathophysiology of OTS remain unclear. Theories involving the autonomic, inflammatory, antioxidative, energy-balance, immune and hormonal systems have clarified some aspects of OTS, but could not explain the complete pathophysiology, ${ }^{1}$ or identify accurate markers of OTS. Proposed markers, including increased plasma glutamine, maximum lactate levels and altered CK reaction to unusual exercise, have been reported in subjects with OTS and related conditions $^{127815182526}$; however, these showed low accuracy for differentiation of healthy and affected athletes, and were not confirmed by further studies. Conversely, impaired hormonal responses to stress induced by maximal exercise have been demonstrated in athletes with OTS, ${ }^{1}{ }^{10}{ }^{12-14}$ and could be precisely distinguished from responses in healthy athletes. However, the reduced hormonal responses to exercise-induced stress verified in these studies could have resulted from impaired signalling from the neuromuscular and cardiovascular systems to the hypothalamus and pituitary, or differences in performance during the stimulation tests. ${ }^{1012-14}$ No studies have identified hormonal changes in OTS as the primary dysfunction. Therefore, efforts to identify tools to improve the diagnosis of OTS have been largely unsuccessful, ${ }^{1}$ 7-18 2526 likely owing to challenges in the methodology design of studies on OTS. The most important challenges include: (i) the selection process; (ii) correct diagnosis of OTS; (iii) the study population and sport(s) modalities performed; (iv) proper exclusion of confounding factors; (v) characteristics of the control group(s), with the use of a second control group of non-athletes; (vi) tests and procedures to be performed and (vii) the use of natural-occurring actual OTS, not induced OTS, which tends to manifest as over-reaching.

The improvement of the methodological design in the EROS study ${ }^{21-24}$ to address these challenges, including the simultaneous analysis of multiple aspects, including basal and stimulated hormones, biochemical data and clinical 
Table 4 Biochemical markers altered in overtraining syndrome

\begin{tabular}{|c|c|}
\hline Altered markers & $\begin{array}{l}\text { Level of alteration (overt, relative or } \\
\text { exacerbated)* }\end{array}$ \\
\hline \multicolumn{2}{|l|}{ Insulin tolerance test (ITT) } \\
\hline $\begin{array}{l}\text { Cortisol during hypoglycaemia in response to ITT }(\mu \mathrm{g} / \mathrm{dL}) \\
\text { Cortisol } 30^{\prime} \text { after hypoglycaemia in response to ITT }(\mu \mathrm{g} / \mathrm{dL}) \\
\text { - Cortisol increase during ITT }(\mu \mathrm{g} / \mathrm{dL})\end{array}$ & Relative \\
\hline $\begin{array}{l}\text { ACTH } 30^{\prime} \text { after hypoglycaemia in response to ITT (pg/mL) } \\
\text { ACTH change during ITT (pg/mL) }\end{array}$ & Overt \\
\hline $\begin{array}{l}\text { Growth hormone }(\mathrm{GH}) \text { during hypoglycaemia in response to ITT ( } \mu \mathrm{g} / \mathrm{L}) \\
\text { GH 30' after hypoglycaemia in response to ITT }(\mu \mathrm{g} / \mathrm{L})\end{array}$ & Relative \\
\hline $\begin{array}{l}\text { Prolactin during hypoglycaemia in response to ITT }(\mathrm{ng} / \mathrm{mL}) \\
\text { Prolactin } 30^{\prime} \text { after hypoglycaemia in response to ITT }(\mathrm{ng} / \mathrm{mL}) \\
\text { Change in prolactin during ITT }(\mathrm{ng} / \mathrm{mL})\end{array}$ & Relative \\
\hline \multicolumn{2}{|l|}{ Basal hormones } \\
\hline Basal GH ( $\mu \mathrm{g} / \mathrm{L})$ & Relative \\
\hline Basal prolactin $(\mu \mathrm{g} / \mathrm{mL})$ & Relative \\
\hline Oestradiol (pg/mL) & Overt \\
\hline Total testosterone (ng/dL) & Relative \\
\hline Testosterone-to-oestradiol ratio & Overt \\
\hline \multicolumn{2}{|l|}{ Other hormones } \\
\hline Total catecholamines ( $\mu \mathrm{g} / 12$ hours) & Exacerbated \\
\hline Nocturnal urinary norepinephrine ( $\mu \mathrm{g} / 12$ hours) & Overt \\
\hline $30^{\prime}$ after awakening salivary cortisol (ng/dL) & Relative \\
\hline \multicolumn{2}{|l|}{ Immunological and muscular markers } \\
\hline Creatine kinase (U/L) & Exacerbated \\
\hline Lactate (nmol/L) & Relative \\
\hline Lymphocytes (/mm³) & Relative \\
\hline Neutrophils $\left(/ \mathrm{mm}^{3}\right)$ & Relative \\
\hline Platelet to lymphocyte ratio & Relative \\
\hline
\end{tabular}

Relative: Unaltered when compared with general population, but altered when compared with healthy athletes.

Exacerbated: Changes normally observed in healthy athletes that are exacerbated in the presence of overtraining syndrome.

*Overt: Markedly and exclusively altered in overtraining syndrome, when compared with both the healthy athletes and the healthy nonphysically active subjects.

ACTH, adrenocorticotropic hormone.

aspects, concurrent comparisons with non-athletes and a relatively large number of athletes, probably the largest number of participants among studies in OTS, ${ }^{10}$ which strengthen our data, resulted in the observation of unexpected similarities between athletes with OTS and NPAC and differences between these groups and healthy athletes. These observations, along with the scenarios, which were only feasible because of the context of the findings in OTS, and the other behaviours shown by the evaluated parameters, allowed us to propose new risk factors, to predict the occurrence of OTS and to hypothesise a new explanation for OTS, and to understand the adaptive physiological responses of healthy athletes better.

\section{Clinical, metabolic and biochemical behaviors in OTS}

In the present analysis, our objective was to understand the context of the five patterns of behaviours observed in the primary findings, and gain new insights on the development and diagnosis of OTS. Most of the non-similar markers between OTS, ATL and NPAC (32/44; 72.7\%) reflected losses of conditioning or adaptive processes that athletes typically undergo, or 'deconditioning'. The most remarkable markers of 'deconditioning' identified were ${ }^{21-24}$ : (i) blunted and late $\mathrm{GH}$, cortisol and prolactin responses to an exercise-independent central stimulation test, which may explain the loss of pace and performance during OTS; (ii) reduced testosterone levels, which may explain the loss of muscle mass in athletes with OTS; (iii) increased oestradiol levels without a concurrent increase in testosterone (reduced testosterone-to-oestradiol ratio), which could cause some of the psychological and body metabolism and composition patterns found in OTS; (iv) worse mood states (particularly fatigue, vigour, depression and confusion), likely correlated with worse sleep quality and hormonal dysfunctions and (v) changes in body metabolism and composition (lower 
metabolic rate and fat burning, more body fat, less muscle mass and less hydration), likely due to a hormonal environment that leads to a muscle-specific catabolic state, and reduction of overall metabolism.

Two specific markers (ie, CK and total catecholamines) indicated an exacerbation of adaptive changes observed in athletes. CK might have resulted from impaired and prolonged muscle recovery, and total catecholamines might have resulted from an attempt to maintain function despite energy depletion. Stimulation of catecholamines might be the shortest and fastest efferent pathway to stimulate the recruitment and availability of energy in an energy-deprived environment, through optimisation of lipolysis, proteolysis and gluconeogenesis in the absence of glycogen and glucose storage.

Seven markers were observed exclusively in OTS $(15.9 \%$ of non-similar parameters), including ACTH response patterns to stimulation, libido, oestradiol and norepinephrine. These markers can be considered specific markers of OTS, without the need for any adaptations to the standard normal range. Conversely, $38.8 \%$ of the selected parameters (26 out of 67 markers) were unaffected by OTS, compared with those typical of athletes or both the athletes and non-athletes, and these parameters most likely have no role in the pathophysiology of OTS.

In short, the finding that approximately two-thirds of the markers underwent physiological adaptations to exercise, the prevailing behaviour observed in OTS were the multiple decrements in athletes' performance in response to these adaptive changes, which were found in a broad range of parameters, including basal and stimulated hormones, psychological patterns, and body metabolism and composition. The second most observed behaviour was the lack of alterations, while few were present or exacerbated only in OTS.

\section{A new hypothesis for the pathophysiology of OTS A new hypothesis for OTS}

All the data obtained from the EROS study reinforce that adaptive changes occurring in athletes (compared with sedentary participants), are at least partially lost during OTS, as a sort of 'mix of deconditioning processes', ${ }^{21-24}$ wherein the results were similar to those of sedentary participants than to those of healthy athletes. Athletes with OTS do not present absolute altered parameters, but show relative dysfunctional responses compared with healthy athletes. The hormonal and metabolic conditioning processes that athletes undergo could explain the progressive improvement of physical performance, while the losses of these conditioning processes that we observed in OTS could be the cause of the paradoxical loss of performance, which is the hallmark of OTS, and not previously understood.

Furthermore, exacerbated 'deconditioning' processes and exclusive findings in OTS could explain the harmful psychological and physical effects observed in individuals with OTS, who lost the benefits of exercising and tended to develop overt and severe issues, particularly in the psychological aspects.
These insights show that we should not usually expect overt biochemical alterations in athletes suspected for OTS, as parameters are usually within the normal range. However, we need to elucidate the particular ranges of each parameter that are to be expected in athletes, as these adapted ranges should be used to analyse athletes at high risk or suspected for OTS. Although we proposed some specific and adapted ranges for the population of athletes, those may be helpful to predict athletes that would further develop OTS, more studies are needed to confirm the consistency of these ranges.

When the results of the EROS study were analysed, ${ }^{21-24}$ we found that none of the markers evaluated in the EROS study could individually trigger or explain the occurrence of OTS, since none were present in all affected athletes. Each affected athlete showed a unique combination of factors that caused OTS. Thus, OTS likely results from different synergistic combinations and interactions of negative factors, which are unique to each athlete, rather than specific triggers or a single combination of factors for all athletes. Hence, aside from the methodological challenges to research on OTS, the absence of clear findings, inconsistent results between healthy athletes and the general population, and the wide range of individual combinations of triggers, risk factors and patterns of OTS could explain the previous inconclusive findings and unsuccessful attempts to elucidate the mechanisms of OTS.

The combination of altered parameters in OTS, unique to each affected athlete, and the absence of a ubiquitously present alteration allow us to predict that there should not be one single precise marker for OTS. Instead, combinations of those markers that better distinct OTS-affected from healthy athletes should be the most reliable type of assessment of OTS.

Also, the present analysis of the findings of the EROS study allowed us to hypothesise that athletes affected by OTS experience a 'hyporesponsive' and 'hypometabolic' state, based on the findings of decreased basal metabolic rate and fat burning, ${ }^{23}$ paradoxical adipocyte saving in fat storages, ${ }^{23}$ impaired hormonal responses to demands, ${ }^{21} 22$ and decreased testosterone, testosterone-to-oestradiol ratio and GH, and increased catecholamines. ${ }^{22} 24$ These findings suggest an antianabolic and procatabolic environment, a global reduction in metabolic pathways and responses, possibly to maximise energy saving and prevent unnecessary energy expenditure, avoid the use of energy storage, and redirect energy for essential pathways, as a major protective mechanism against long-term starvation-like circumstances. Thus, indirect markers of altered metabolism, such as abnormal body fat gain, paradoxical muscle loss and worse libido or mood states (resulted from reduced testosterone), may be early indicators of imminent OTS, even prior to altered biochemical results or decreased performance.

A total of 5 of the 50 excluded parameters were unsuitable for the present analysis because they were predictors that directly depended on participants' habits, but were not influenced by their physical activity or the presence of OTS. These included eating patterns and concurrent 


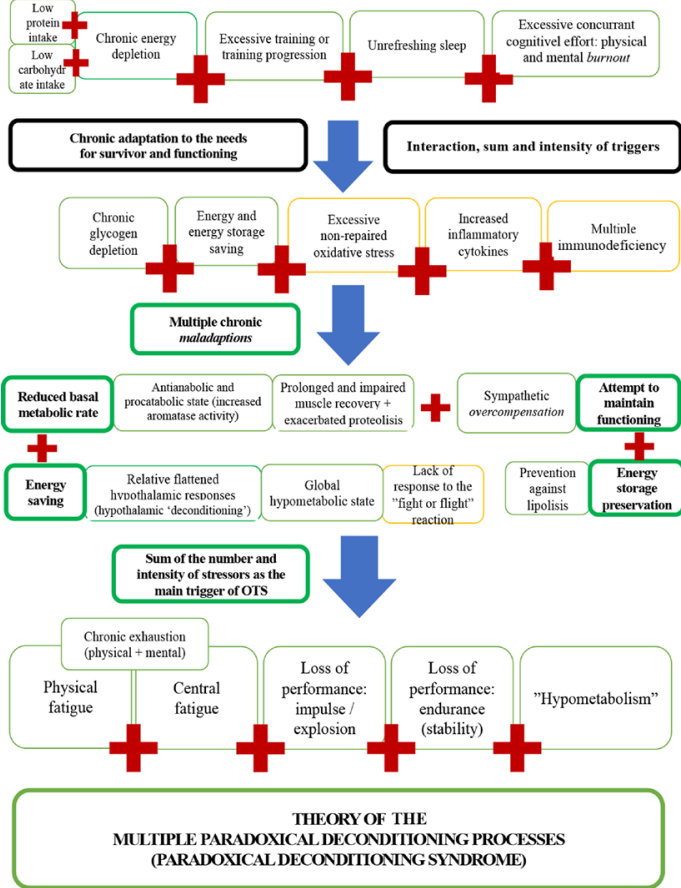

Figure 4 Triggers, pathophysiology and consequences of overtraining syndrome (OTS).

cognitive effort. Among these parameters, we identified the following risk factors or predictors of $\mathrm{OTS}^{23}$ : (i) mean number of daily hours of work/study $>8$ hours / day; (ii) carbohydrate intake $<5.0 \mathrm{~g} / \mathrm{kg} /$ day; (iii) protein intake $<1.6 \mathrm{~g} / \mathrm{kg} /$ day and (iv) total daily calorie intake $<35 \mathrm{kcal} / \mathrm{kg} /$ day ${ }^{23}$ Fat intake $(\mathrm{g} / \mathrm{kg} /$ day) did not predict OTS. ${ }^{23}$ This reinforces the theory that OTS rises from unique combinations of risk factors and stressors, rather than excessive training alone.

In summary, OTS was demonstrated to be a clinical state, which is induced by a distinctive combination of chronic stressors, leading to several synergistic combinations of metabolic, hormonal, inflammatory, immunological, neurological, cardiovascular and psychological dysfunctions that cause a dysfunctional 'hypometabolic' and 'hyporesponsive' state. The blunted responses to demands are due to multiple failures to achieve the beneficial effects of conditioning that athletes typically experience.

Together, the new findings on OTS allowed us to propose that a comprehensive analysis of the athlete, including the evaluation for risk factors, early clinical signs, and relatively altered biochemistry and hormones, should be the most effective approach for the prevention of imminent OTS, prior to the occurrence of decreased performance.

The triggers, risk factors, proposed pathophysiology and consequences of OTS, and the novel hypotheses derived from the novel markers identified in the EROS study are shown in figure 4 . A predictive model with practical applications for the prevention and early detection of OTS, built from the insights of the EROS study, is disclosed in figure 5 .

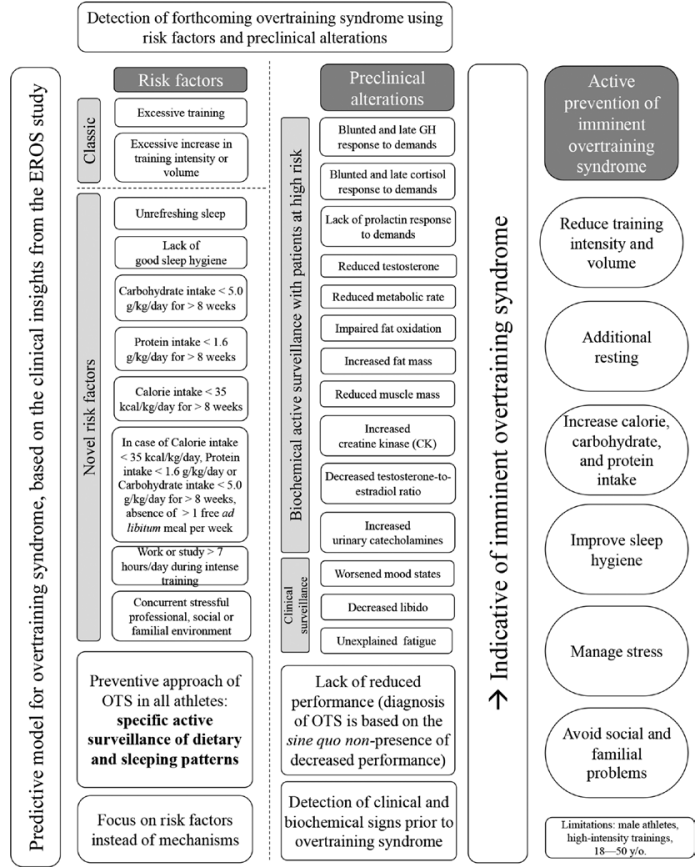

Figure 5 Predictive model for the identification of overtraining syndrome (OTS). EROS, Endocrine and Metabolic Responses on Overtraining Syndrome; GH, growth hormone; $y / o$, years old.

However, larger studies are required to confirm our hypothesis. We could not make firm conclusions regarding the physiopathology and clinical and biochemical behaviours in OTS based on our data, but could propose a plausible hypothesis to accurately explain OTS. Studies published after our primary findings reinforce our hypothesis. ${ }^{27-29}$ Our data could form the basis of further studies in larger groups to confirm our hypothesis.

\section{'Paradoxical deconditioning syndrome': renaming 'overtraining syndrome'}

The term 'overtraining syndrome' was coined because of the initial idea that excessive training caused the clinical features of OTS. However, our data showed that underperformance (loss of physical performance), the hallmark of OTS, is due to an extensive number of deconditioning processes, caused by unique combinations of clinical and social risk factors.

Our data suggest that OTS is not a simple consequence of 'overtraining', but resulted from a combination of different risk factors and harmful clinical behaviours, among which some were unprecedentedly recognised, including insufficient caloric, protein or carbohydrate intake, bad sleep quality and excessive concurrent cognitive effort. The most remarkable aspect of OTS is, therefore, not 'overtraining', but a blend of conditioning shortfalls, or 'unexpected and paradoxical deconditioning'. The loss of multiple conditioning processes naturally leads to a loss in the physical conditioning, clinically observed as reduced performance. On the basis of the evidence shown in the present study, in which the 
data is likely stronger than previous data, we suggest that the term 'paradoxical deconditioning syndrome', is more representative of the key aspects of this syndrome.

\section{CONCLUSIONS}

The predominant marker patterns observed in OTS were different from those in healthy athletes but similar to sedentary controls, suggesting that OTS is mostly a mix of deconditioning processes, independent of excessive training and triggered by additional risk factors, previously unrecognised, including lack of sufficient caloric intake, fair sleep quality and concurrent cognitive effort. The characterisation of OTS as a combination of multiple decrements in performance in response to conditioning processes in athletes is more representative of an essential clinical aspect of the syndrome-decrease in performance. We found sufficient data to suggest that 'paradoxical deconditioning syndrome of the athlete' is a more appropriate and descriptive name for OTS than the previous misnomer, which is focused only on excessive training. Further studies are required to elucidate the mechanisms of OTS revealed by the EROS study.

Contributors FAC and CEK developed the central idea of the present manuscript. FAC performed the tests of the EROS study, compilated the data, analysed the results and participated in the discussions. CEK actively participated in the discussion, supervised and reviewed the results, helped with the final version of the manuscript and gave the last word before the submission.

Funding The authors have not declared a specific grant for this research from any funding agency in the public, commercial or not-for-profit sectors.

Disclaimer No funding was provided for the study, as the materials were directly provided by: (1) DASA Diagnostics (São Paulo, SP, and Brasília, DF, Brazil), (2) Federal University of São Paulo (São Paulo, SP, Brazil) and (3) Corpometria Institute (Brasília, DF, Brazil).

Competing interests The results of the present study do not constitute an endorsement by ACSM. The authors declare no competing interests, including no professional relationships with companies or manufacturers who will benefit from the results of the present study. We did not directly involve patient and public (PPI) in this study, but the database used in the study was developed together with PPI, and a personalised feedback was given based on participant's expectations.

Patient consent for publication Not required.

Ethics approval The study was approved by the ethical committee of the Federal University of São Paulo (approval number: 1093965).

Provenance and peer review Not commissioned; externally peer reviewed.

Open access This is an open access article distributed in accordance with the Creative Commons Attribution Non Commercial (CC BY-NC 4.0) license, which permits others to distribute, remix, adapt, build upon this work non-commercially, and license their derivative works on different terms, provided the original work is properly cited, appropriate credit is given, any changes made indicated, and the use is non-commercial. See: http://creativecommons.org/licenses/by-nc/4.0/.

\section{REFERENCES}

1. Meeusen R, Duclos M, Foster C, et al. Prevention, diagnosis, and treatment of the overtraining syndrome: joint consensus statement of the European College of Sport science and the American College of sports medicine. Med Sci Sports Exerc 2013;45:1-24.

2. Rietjens GJWM, Kuipers H, Adam JJ, et al. Physiological, biochemical and psychological markers of strenuous traininginduced fatigue. Int J Sports Med 2005;26:16-26.

3. Furth HG, Youniss J. Effect of overtraining of three discrimination shifts in children. Journal of Comparative and Physiological Psychology 1964;57:290-3.
4. Smit PJ. Sports medicine and 'overtraining'. S Afr Med J 1978;54.

5. McTernan EJ, Leiken AM. A pyramid model of health manpower in the 1980s. J Health Polit Policy Law 1982;6:739-51.

6. Raglin J, Sawamura S, Alexiou S, et al. Training practices and Staleness in 13-18-Year-Old swimmers: a cross-cultural study. Pediatric Exercise Science 2000;12:61-70.

7. Coutts A, Reaburn P, Piva T, et al. Changes in selected biochemical, muscular strength, power, and endurance measures during deliberate overreaching and tapering in Rugby League players. Int $J$ Sports Med 2007;28:116-24.

8. Nederhof E, Zwerver J, Brink M, et al. Different diagnostic tools in nonfunctional Overreaching. Int J Sports Med 2008;29:590-7.

9. Slivka DR, Hailes WS, Cuddy JS, et al. Effects of 21 days of intensified training on markers of Overtraining. Journal of Strength and Conditioning Research 2010;24:2604-12.

10. Cadegiani FA, Kater CE. Hormonal aspects of overtraining syndrome: a systematic review. BMC Sports Sci Med Rehabil 2017;9.

11. Coutts AJ, Reaburn P, Piva TJ, et al. Monitoring for overreaching in Rugby League players. Eur J Appl Physiol 2007;99:313-24

12. Meeusen $R$, Nederhof E, Buyse L, et al. Diagnosing overtraining in athletes using the two-bout exercise protocol. Br J Sports Med 2010;44:642-8.

13. Meeusen R, Piacentini MF, Busschaert B, et al. Hormonal responses in athletes: the use of a two bout exercise protocol to detect subtle differences in (over)training status. Eur J Appl Physiol 2004;91:140-6.

14. Urhausen A, Gabriel HH, Kindermann W. Impaired pituitary hormonal response to exhaustive exercise in overtrained endurance athletes. Med Sci Sports Exerc 1998;30:407-14.

15. Koch AJ, Pereira R, Machado M. The creatine kinase response to resistance exercise. J Musculoskelet Neuronal Interact 2014;14:68-77.

16. Lehmann M, Schnee W, Scheu R, et al. Decreased Nocturnal Catecholamine Excretion: Parameter for an Overtraining Syndrome in Athletes?*. Int J Sports Med 1992;13:236-42.

17. Fry AC, Kraemer WJ, Ramsey LT. Pituitary-adrenal-gonadal responses to high-intensity resistance exercise overtraining. Journal of Applied Physiology 1998;85:2352-9.

18. Hug M, Mullis PE, Vogt $M$, et al. Training modalities: over-reaching and over-training in athletes, including a study of the role of hormones. Best Practice \& Research Clinical Endocrinology \& Metabolism 2003;17:191-209.

19. Nederhof E, Lemmink KAPM, Visscher C, et al. Psychomotor speed: possibly a new marker for overtraining syndrome. Sports Med 2006;36:817-28.

20. Stamford B. Avoiding and recovering from Overtraining. The Physician and Sportsmedicine 1983;11.

21. Cadegiani FA, Kater CE, Hypothalamic-pituitary-adrenal KCE. Hypothalamic-Pituitary-Adrenal (HPA) Axis Functioning in Overtraining Syndrome: Findings from Endocrine and Metabolic Responses on Overtraining Syndrome (EROS) - EROS-HPA Axis. Sports Medicine - Open 2017;3.

22. Cadegiani FA, Kater CE, Hormone G. Hormonal response to a non-exercise stress test in athletes with overtraining syndrome: results from the endocrine and metabolic responses on Overtraining syndrome (EROS) - EROS-STRESS. Journal of Science and Medicine in Sport 2018;21:648-53.

23. Cadegiani FA, Kater CE. Body composition, metabolism, sleep, psychological and eating patterns of overtraining syndrome: results of the EROS study (EROS-PROFILE). Journal of Sports Sciences 2018;36:1902-10.

24. Cadegiani FA, Kater CE. Basal hormones and biochemical markers as predictors of OTS: results from the Endocrine and metabolic Responses on Overtraining Syndrome (EROS) study - EROS-BASAL. $J$ Athl Training. Acceptedln press.

25. Kreher JB, Schwartz JB. Overtraining syndrome: a practical guide. Sports Health 2012;4:128-38.

26. LEHMAN M, Foster C, Keul J. Overtraining in endurance athletes: a brief review. Medicine \& Science in Sports \& Exercise 1993;25:854-62.

27. Woods AL, Rice AJ, Garvican-Lewis LA, et al. The effects of intensified training on resting metabolic rate (RMR), body composition and performance in trained cyclists. Plos One 2018;13:e0191644.

28. Viana RB, Gentil P, Lorenço VS, et al. Identifying the predisposing factors, signs and symptoms of overreaching and overtraining in physical education professionals. PeerJ 2018;6:e4994.

29. Pontzer H. Energy constraint as a novel mechanism linking exercise and health. Physiology 2018;33:384-93. 